\title{
COMPTAGE DE STRIES SUR ALLIAGES LÉGERS ROMPUS EN FATIGUE
}

\author{
J. ODORICO et $\mathrm{Ph}$. LE GALL (*)
}

S. N. I. A. S. 12, rue Pasteur, 92150 Suresnes, France

\begin{abstract}
Résumé. - Des comptages systématiques de stries de fatigue des éprouvettes rompues de type IRWIN en A-U4G1 T 3, d'épaisseur $e=2 \mathrm{~mm}$, en A-U4G1 T $351 e=1,5 \mathrm{~mm}$, en A-U2GN T 651 $e=1,5 \mathrm{~mm}, e=3 \mathrm{~mm}$ et $e=5 \mathrm{~mm}$ nous ont permis de trouver des corrélations entre les vitesses macroscopiques de fissuration et les distances moyennes entre les stries. Ces corrélations dépendent légèrement de l'épaisseur et sont valables pour des vitesses de fissuration $\mathrm{d} a / \mathrm{d} N$ allant de $0,05 \mu /$ cycle à $0,5 \mu /$ cycle. De ces résultats nous pouvons déduire des relations entre :

- le nombre de stries et le nombre de cyclage de contrainte,

- les distances moyennes entre les stries et la contrainte maxi appliquée pendant la propagation.
\end{abstract}

Abstract. - Experimental measurement of fatigue striation spacing on centrally notched fracture surface of AU4G1-T 3 specimens, $2 \mathrm{~mm}$ thick, AU4G1-T 351, $1.5 \mathrm{~mm}$ thick, AU2GN-T 651, $1.5,3$ and $5 \mathrm{~mm}$ thick have been realized. We have found some correlation between the macroscopic crack growth rate and the average value of striation spacing. This correlation depends slightly on the thickness and are available for crack growth rate $\mathrm{d} a / \mathrm{d} N$ from $0.05 \mu /$ cycle to $0.5 \mu /$ cycle.

From these results we can obtain the relation between :

- the number of striation spacings and the number of load cycles,

- the average striation spacing and the imposition of peak loads in the loading sequence.

1. Introduction. - En vue d'apporter un renseignement plus complet aux Bureaux d'Etudes et aux Services Après-Vente par les expertises des pièces en alliages légers rompues en fatigue, nous avons entrepris, au Laboratoire Central de l'Aérospatiale, l'étude de la mesure de la vitesse de propagation de ce type de fissure.

Cette vitesse devant pouvoir se "lire » sur la surface de rupture, nous sommes partis des travaux de R. Pelloux $\left(^{1}\right)$ démontrant clairement qu'une strie de fatigue est marquée par un cyclage en contrainte.

Les premières études remontent déjà à 5 ans environ et récemment, dans le cadre d'un marché DGRST, nous avons complété nos connaissances en s'appuyant surtout sur les représentations maintenant acquises grâce à la mécanique de la rupture.

2. Eprouvettes d'essai. - Dans un premier temps, nous avons constitué un stock d'éprouvettes étalons fissurées en fatigue dont la définition répondait aux souhaits suivants :

- de forme simple permettant de connaître la relation entre $K$ (facteur d'intensité de contrainte), la longueur de crique et la contrainte maximum de sollicitation.

(*) Ingénieurs à la Société Nationale Industrielle Aérospatiale.

(1) J. Corey McMillan et Régis M. N. Pelloux, Laboratoire de Recherche BOEING, Document D 182.0558, juillet 1966.
- de forme dont l'épaisseur est représentative des panneaux de fuselage ou de voilure d'avion.

Nous avons arrêté notre choix sur une éprouvette de type IRWIN sollicitée en traction avec un cycle $\mathrm{F} / 0,1 \mathrm{~F}$ à la fréquence de $20 \mathrm{~Hz}$ en ambiance ordinaire de Laboratoire.

Dans cette éprouvette :

$$
\Delta K=\frac{\Delta \sigma \sqrt{\pi a}}{\sqrt{1-\left(\frac{2 a}{w}\right)^{2}}} .
$$

Les différentes configurations étalonnées ont été les suivantes :

\section{TABLEAU I}

Les essais de fatigue

\begin{tabular}{|c|c|c|c|}
\hline Nuances & Traitement & $e(\mathrm{~mm})$ & $\begin{array}{c}\sigma \operatorname{maxi} \\
\text { pleine tôle }\end{array}$ \\
\hline A-U4Gl & $\begin{array}{l}\text { T } 3 \\
T 351\end{array}$ & $\begin{array}{l}2 \\
1,5\end{array}$ & $\begin{array}{l}\text { de } 10 \text { à } 18 \mathrm{hb} \\
\text { de } 10 \text { à } 16 \mathrm{hb}\end{array}$ \\
\hline A-U2GN & T 651 & $\left.\begin{array}{l}1,5 \\
3 \\
5\end{array}\right\}$ & de 10 à $16 \mathrm{hb}$ \\
\hline
\end{tabular}

Pour chaque valeur de paramètre d'essai, deux éprouvettes, au moins, ont été réalisées et exploitées.

Pour étalonner nos éprouvettes en vitesse de propagation, nous avons suivi celles-ci à l'aide d'un appa- 
reillage automatique ou "suiveur de criques" dont le principe repose sur les courants de Foucault. Chacune des deux extrémités de la crique est suivie par une ferrite dont la position est enregistrée en fonction du temps, lui-même proportionnel au nombre de cycles appliqués à l'éprouvette.

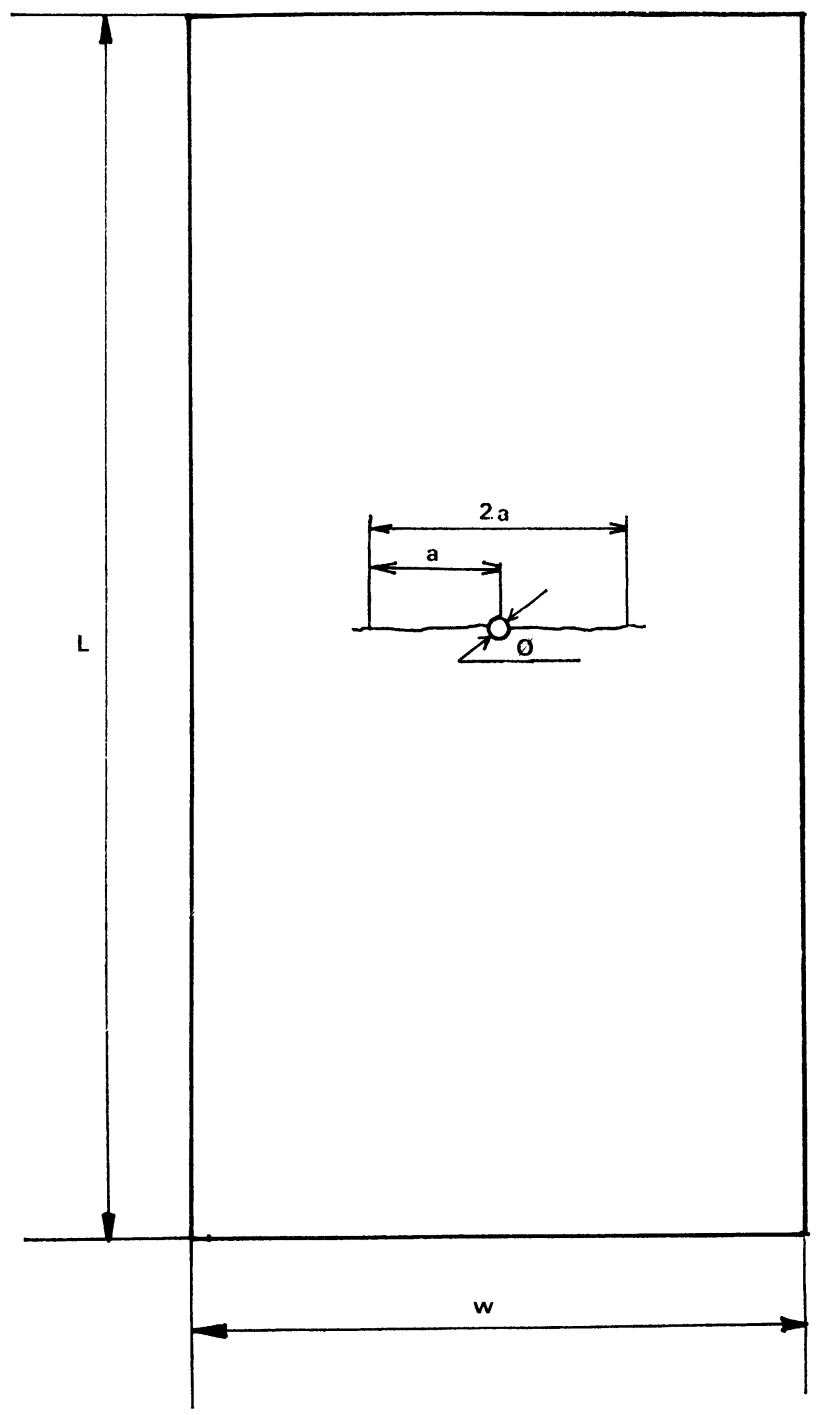

Fig. 1. - Eprouvette de fatigue : $W=150 \mathrm{~mm}, l=300 \mathrm{~mm}$, $\varnothing=2 \mathrm{~mm}$.

Sans entrer dans le détail de fonctionnement de cet appareil, il faut signaler l'un de ses inconvénients majeurs pour nos expériences: il discerne très mal le début de fissuration et c'est seulement pour $a \geqslant 3 \mathrm{~mm}$ que l'enregistrement est sûr.

3. Examen des surfaces de rupture des éprouvettes étalons au microscope électronique à transmission. Nous avons exploité l'ensemble des ruptures étalons ainsi obtenues par l'intermédiaire de réplique carbone obtenue sur réplique primaire en acétate de cellulose.

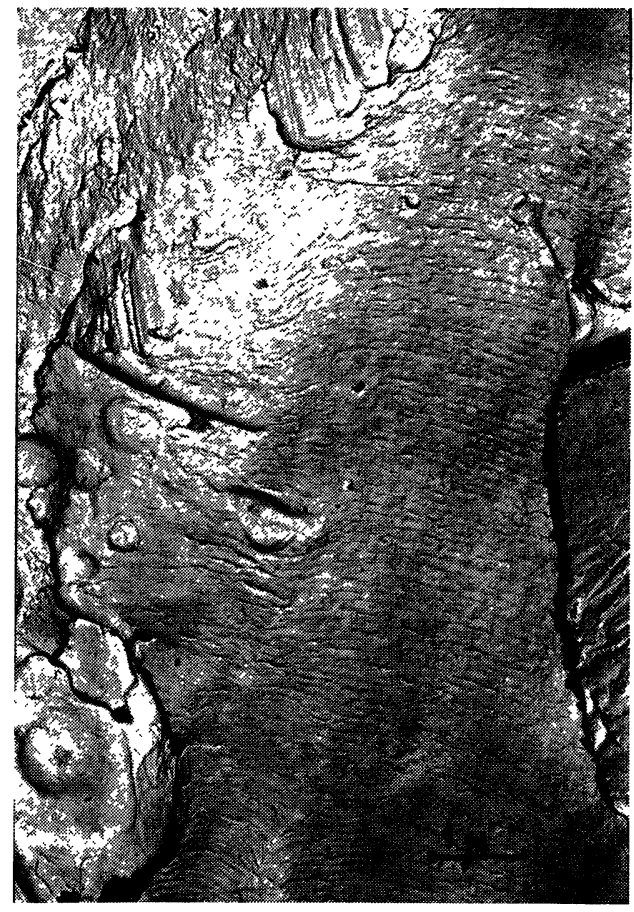

FIG. 2.

Pour «exploiter» des répliques (comptage de stries) nous procédons de la façon suivante :

Sur une réplique unité, de taille environ $1 \times 1 \mathrm{~mm}$, nous recherchons les zones striées en les sélectionnant comme suit :

- zones à stries suffisamment grandes ( $>$ à $10 \mu^{2}$ ),

- zones à stries bien dessinées,

- zones à stries ne comprenant pas de variation locale d'interstrie (sur quelques $\mu$ ), ce qui exclut les zones comprenant des joints de grain ou des précipités.

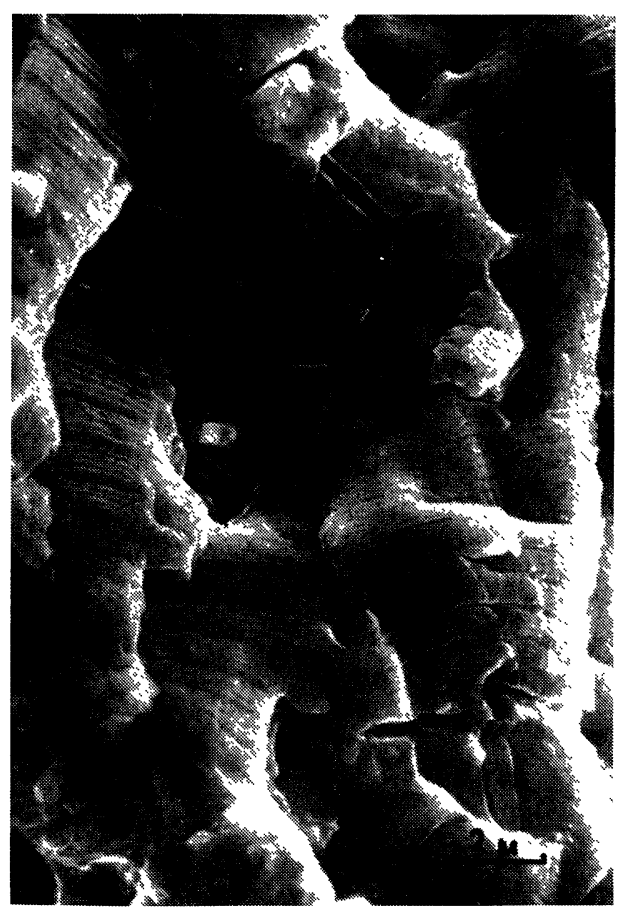

FIG. 3. - «Stries de fatigue vues au M. E. B.». 


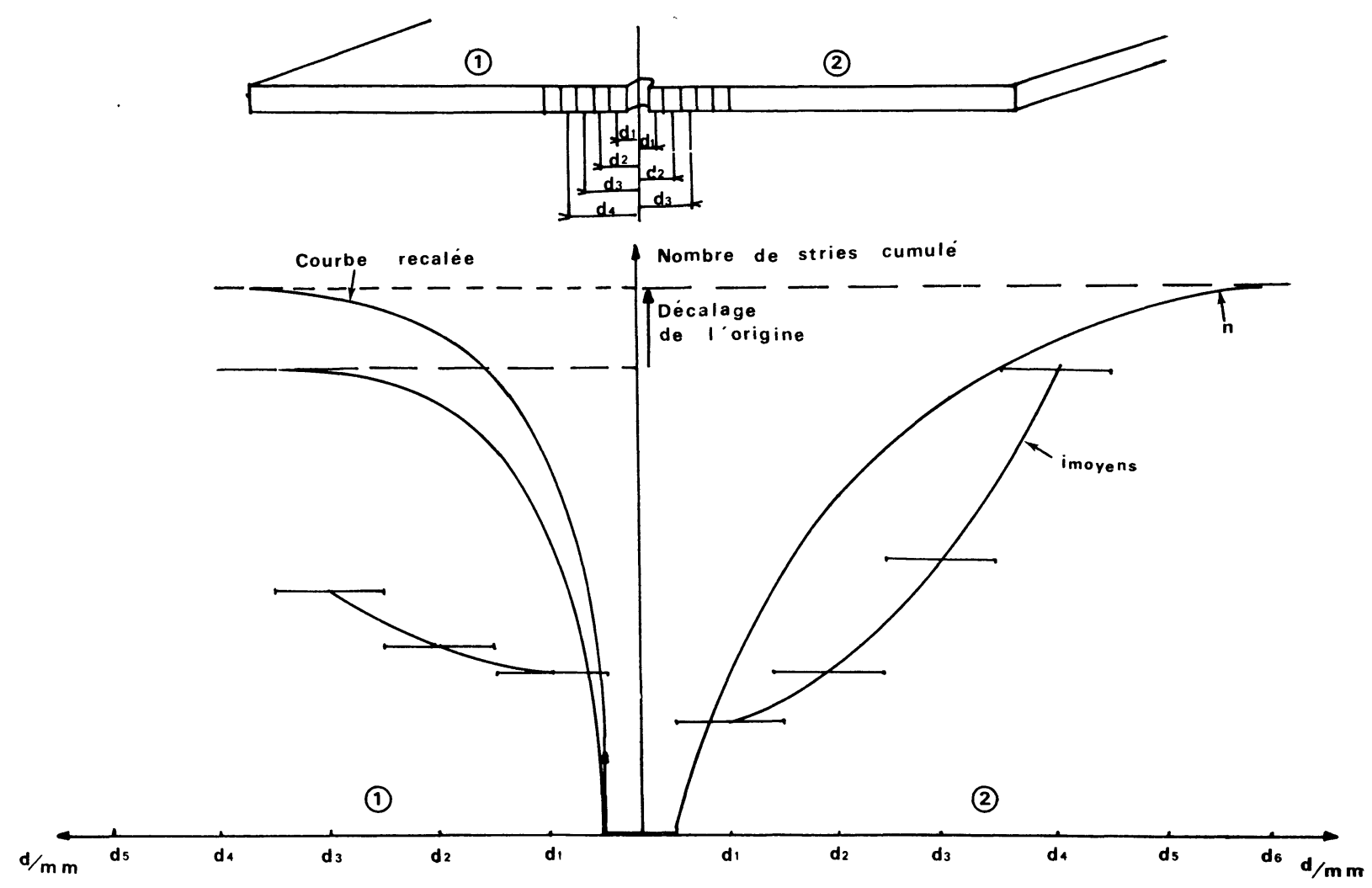

Fig. 4. - Nombre cumulé de stries en fonction de la distance moyenne de la réplique à l'axe de l'entaille. Au-dessus schéma de la prise de réplique sur la rupture.

Ensuite, sur ces zones, nous comptons le nombre de stries par micron de propagation, chaque mesure portant sur environ 8 à 10 stries successives et nous répétons cette mesure 10 à 20 fois sur la réplique unité.

L'ensemble de ces mesures nous permet ainsi de sortir une valeur d'interstrie moyenne valable pour toute la réplique unité.

De cet interstrie moyen, il est également possible de remonter au nombre de stries pour propager la fissure de la largeur de la réplique unité :

$$
n=l / i_{\text {moy }} \text {. }
$$

Ceci permet de donner un nombre de stries pour une propagation donnée.

Dans le cas d'une éprouvette telle que la nôtre, il est fréquent d'avoir des départs dissymétriques (non appréciés par le suiveur de criques) et les courbes comptage de stries des deux côtés de la crique sont recalées pour que les deux côtés de l'éprouvette se rompent dans le même temps.

4. Résultats obtenus. - Sur des graphiques semilogarithmiques, nous avons porté simultanément et pour toutes les valeurs de $\sigma$ maxi essayées les résultats enregistrés pendant la constitution des fissures étalons sur la machine de fatigue et les résultats de comptage en admettant que $\mathrm{d} a / \mathrm{d} n=i_{\text {moy }}$ c'est-à-dire que l'avancement du front de fissure par cyclage de contrainte est égal à l'interstrie moyen trouvé par comptage.
Pour la nuance A-U4G1, qu'elle soit T 3 (trempé, tractionné par redressage et maturé à température ambiante) ou T351 (trempé, traction de $1 \%$ et maturé) en 1,5 et $2 \mathrm{~mm}$, les résultats sont très semblables, l'épaisseur $2 \mathrm{~mm}$ donnant, toutefois, des résultats moins favorables que l'épaisseur $1,5 \mathrm{~mm}$.

Là aussi les résultats sont assez semblables aux précédents pour l'A-U2GN T 651 (trempé, tractionné de $1 \%$ et revenu $22 \mathrm{~h}$ à $190^{\circ} \mathrm{C}$ ). Pourtant une tendance aperçue sur A-U4G1 se distingue parfaitement ici : c'est l'effet de l'épaisseur.

La courbe de comptage de stries se couche d'autant plus que l'épaisseur est forte.

5. Discussion. - En examinant les courbes ciaprès, il ressort clairement que :

- pour les basses valeurs de vitesse macroscopique de la fissure (de 0,1 à 0,5 ou $1 \mu /$ cycle) il y a une très bonne concordance avec l'interstrie moyen mesuré au microscope électronique à transmission.

- pour les valeurs de vitesse macroscopique de la fissure supérieures à $1 \mu /$ cycle, il n'y a plus aucune concordance avec l'interstrie moyen mesuré au microscope électronique à transmission.

En fait, au cours des comptages, les constatations sont les suivantes :

- en début de fissure, le $i_{\text {moy }}$ est petit (de l'ordre de $0,1 \mu$ ) et les plages à stries sont très nombreuses. - au fur et à mesure que l'on s'éloigne du départ de la fissure le $i_{\text {moy }}$ croît, les plages à stries diminuent. 


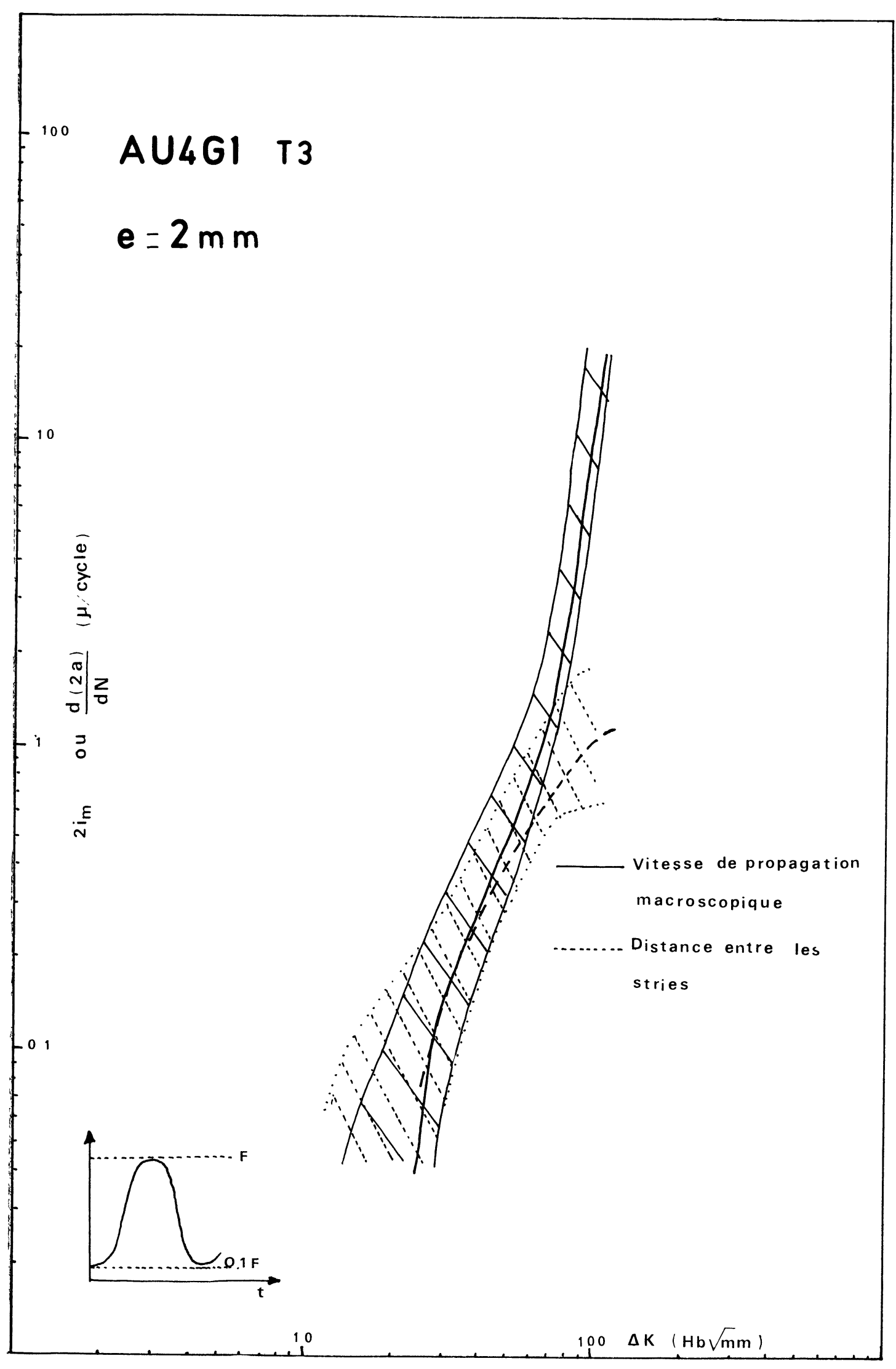

FIg. 5. - Courbes de Paris de l'A-U4G1 T $3 e=2 \mathrm{~mm}$. 


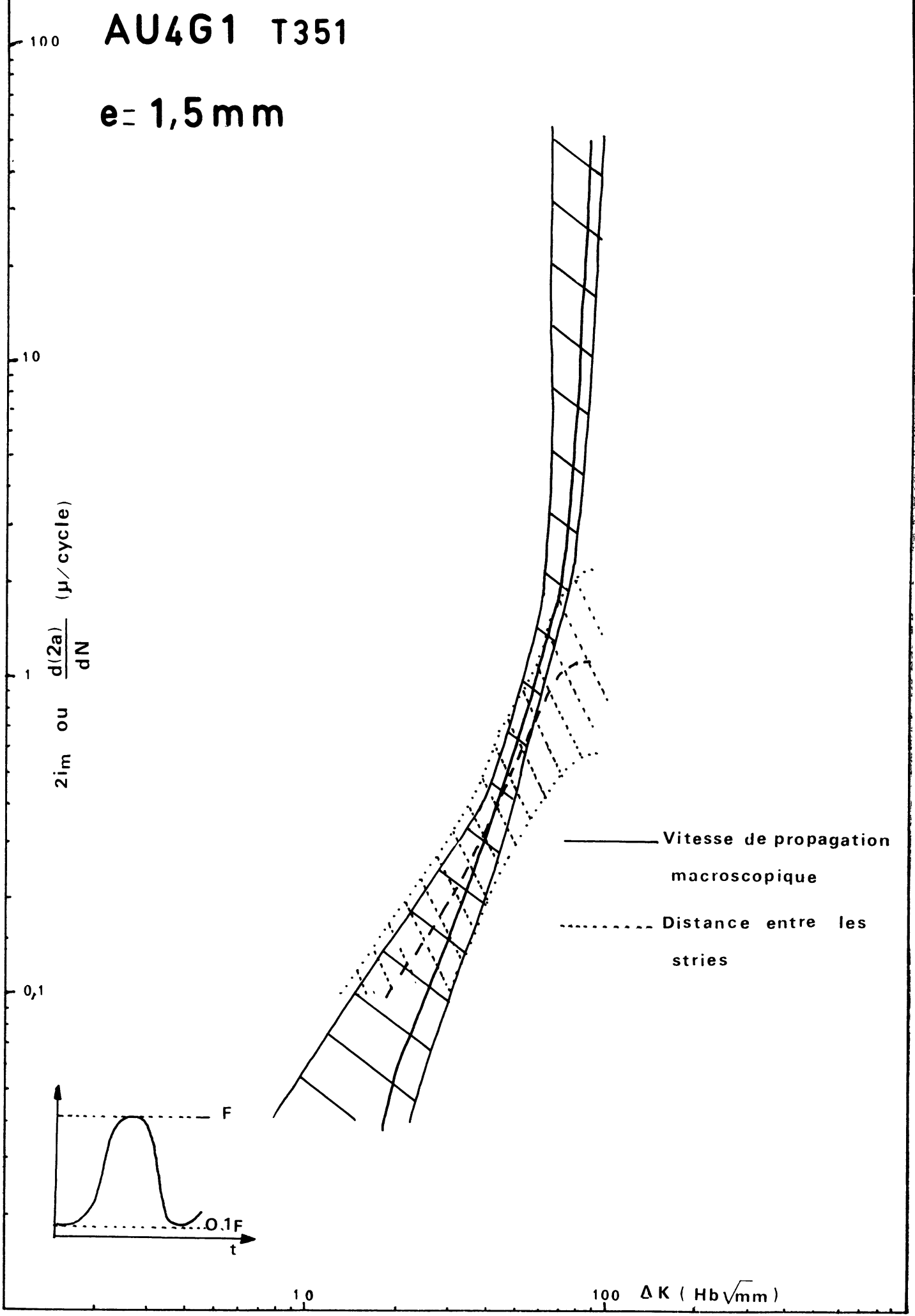

FIG. 6. - Courbes de Paris de l'A-U4G1 T $351 e=1,5 \mathrm{~mm}$. 


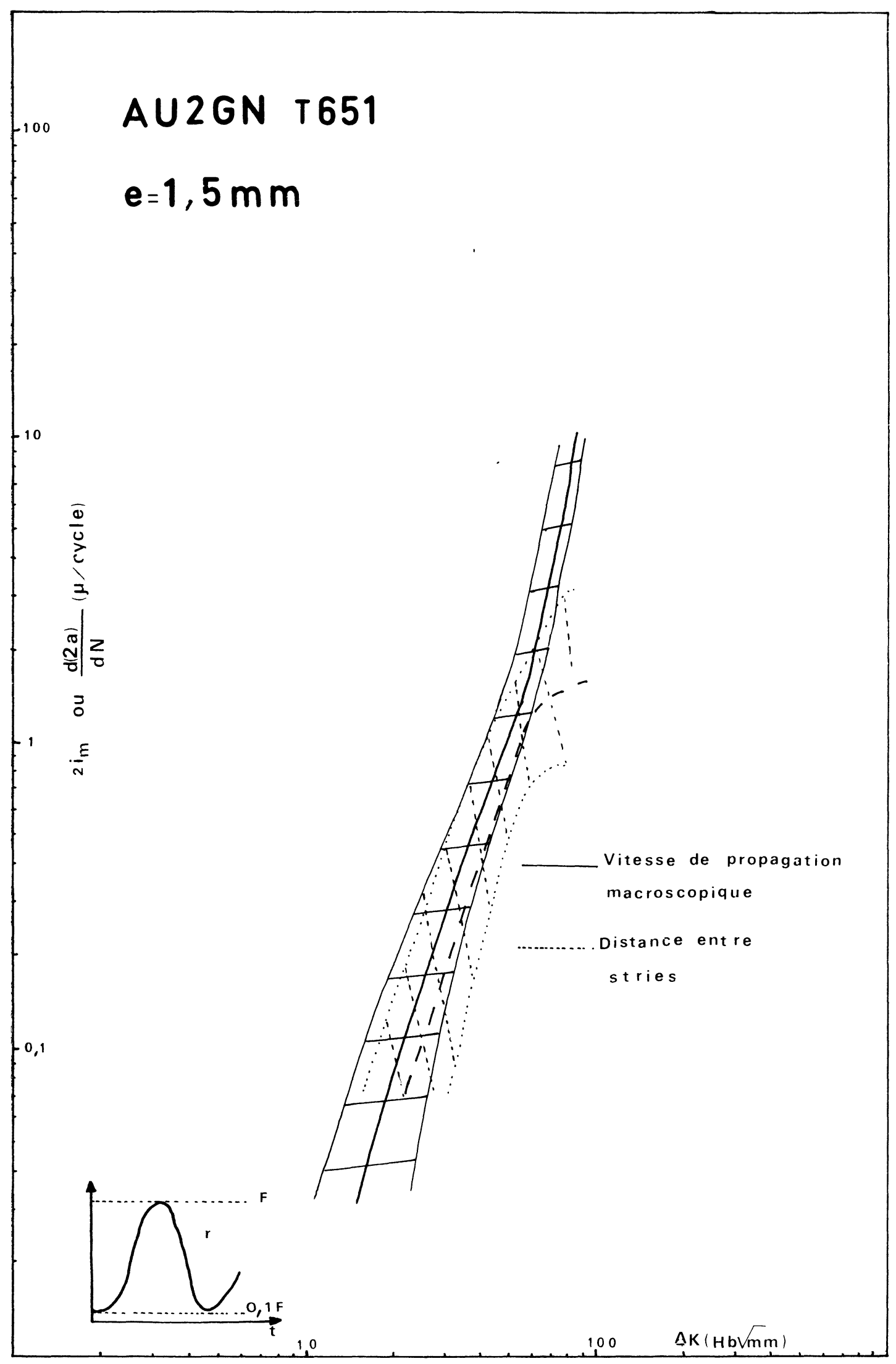

Fig. 7. - Courbes de Paris de l'A-U2GN T $651 e=1,5 \mathrm{~mm}$. 


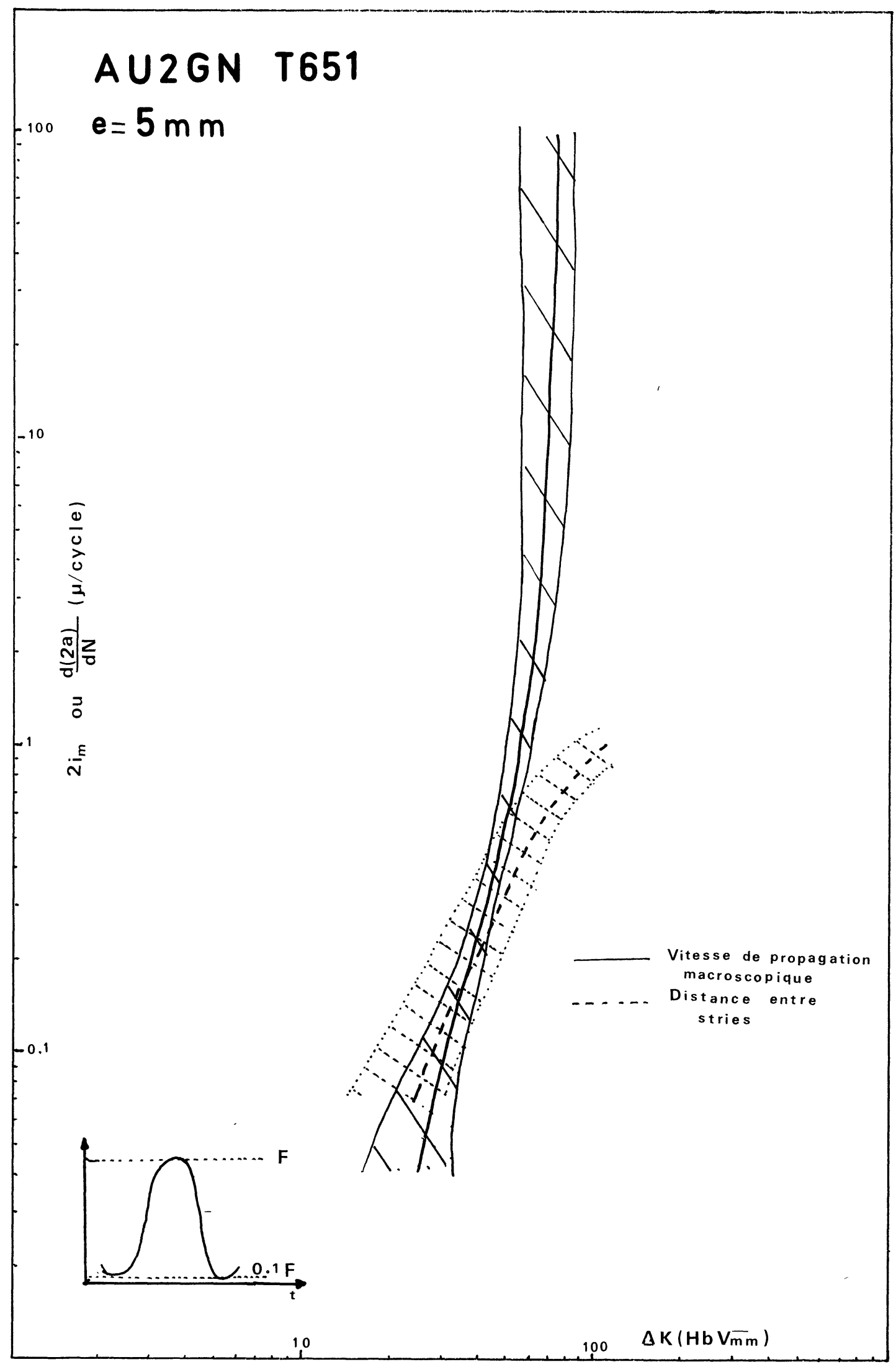

FIG. 8. - Courbes de Paris de l'A-U2GN T $651 e=3 \mathrm{~mm}$. 


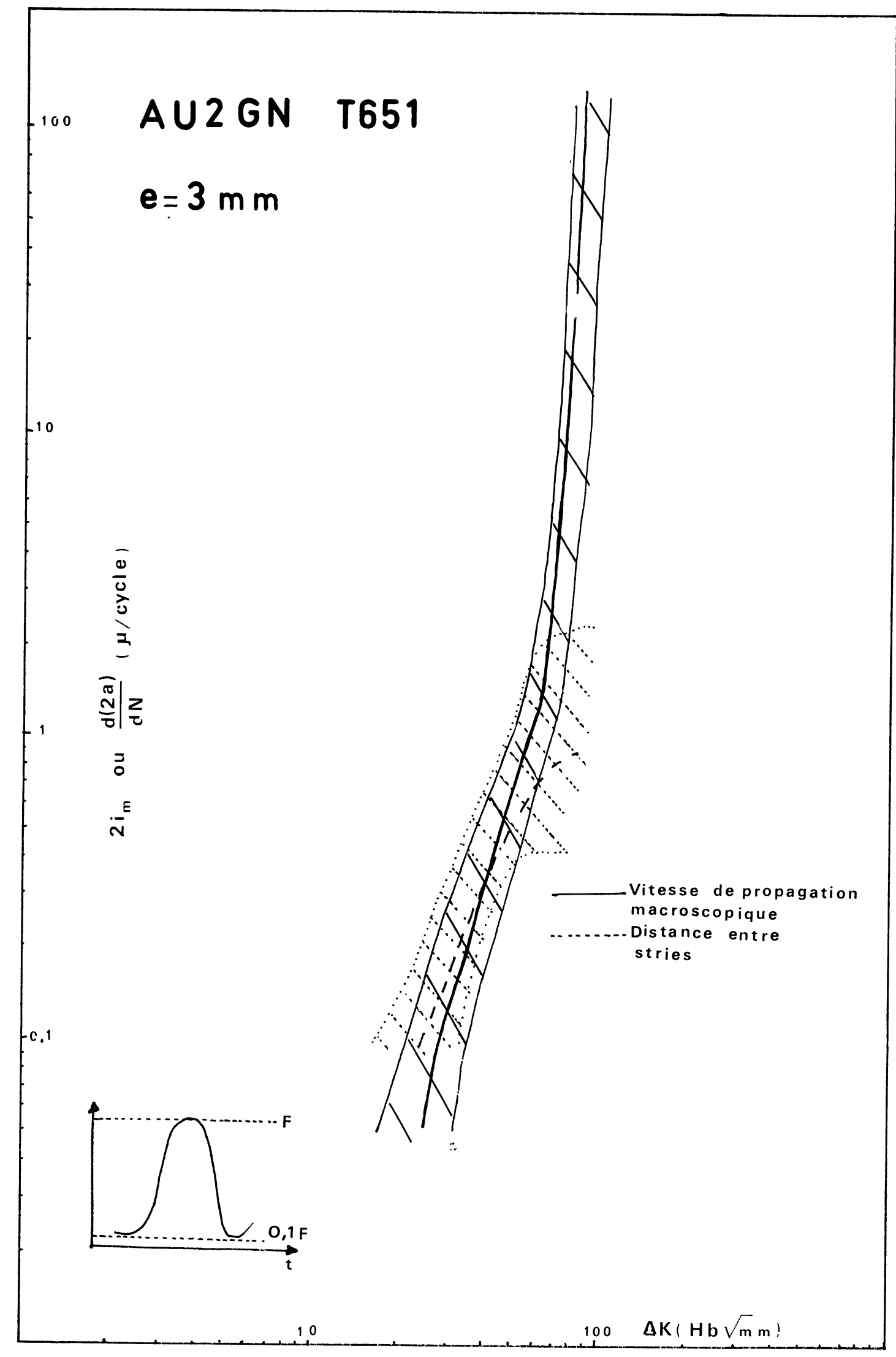

FIG. 9. - Courbes de Paris de l'A-U2GN T $651 e=5 \mathrm{~mm}$. 


\section{TABLEAU II}

Domaines de validité des comptages de stries pour les faibles épaisseurs

\begin{tabular}{|c|c|c|c|c|}
\hline \multirow{2}{*}{ Alliages } & \multirow{2}{*}{$\begin{array}{l}\text { Traitement } \\
\text { т } 3\end{array}$} & \multirow{2}{*}{$\frac{e(\mathrm{~mm})}{2}$} & \multicolumn{2}{|c|}{$\begin{array}{l}\text { Intervalle de vitesse macroscopique } \mathrm{d} a / \mathrm{d} \\
\text { dans lequel la corrélation avec } i_{\text {moy }} \text { est va }\end{array}$} \\
\hline & & & $0,04 \mu /$ cycle & $0,3 \mu /$ cycle \\
\hline A-U4Gl & Т 351 & 1,5 & $\begin{array}{l}\Delta K=25 \mathrm{hb} \sqrt{\mathrm{mm}} \\
0,05 \mu / \text { cycle }\end{array}$ & $\begin{array}{l}\Delta K=60 \mathrm{hb} \sqrt{\mathrm{mm}} \\
0,4 \mu / \text { cycle }\end{array}$ \\
\hline & & 1,5 & $\begin{array}{l}\Delta K=20 \mathrm{hb} \sqrt{\mathrm{mm}} \\
0,05 \mu / \text { cycle }\end{array}$ & $\begin{array}{l}\Delta K=60 \mathrm{hb} \sqrt{\mathrm{mm}} \\
0,5 \mu / \text { cycle } \\
\Delta K=55 \mathrm{hb} \sqrt{\mathrm{mm}}\end{array}$ \\
\hline A-U2GN & T 651 & 3 & $\begin{array}{l}\Delta K=25 \mathrm{hb} \sqrt{\mathrm{mm}} \\
0,04 \mu / \text { cycle }\end{array}$ & $\begin{array}{l}\Delta K=55 \mathrm{hb} \sqrt{\mathrm{mm}} \\
0,25 \mu / \text { cycle }\end{array}$ \\
\hline & & 5 & $\begin{array}{l}\Delta K=25 \mathrm{hb} \sqrt{\mathrm{mm}} \\
0,04 \mu / \text { cycle } \\
\Delta K=25 \mathrm{hb} \sqrt{\mathrm{mm}}\end{array}$ & $\begin{array}{l}\Delta K=50 \mathrm{hb} \sqrt{\mathrm{mm}} \\
0,15 \mu / \text { cycle } \\
\Delta K=45 \mathrm{hb} \sqrt{\mathrm{mm}}\end{array}$ \\
\hline
\end{tabular}

Finalement l'interstrie plafonne à une certaine valeur limite, $i_{\mathrm{e}}$, et la fréquence des zones à stries diminue jusqu'à disparition quasi totale. Nous pensons que, dans ces cas limites, les zones striées correspondent à des ralentissements locaux du front de fissuration. Ces ralentissements, de plus en plus rares, sont propices à la formation des stries tandis que la vitesse de propagation macroscopique croît de manière continue.

Les grandeurs limites des interstries dépendent semble-t-il de la géométrie des pièces fissurées et de la plus ou moins grande complexité du cyclage des contraintes appliquées.

Dans les alliages et les cas étudiés :

- Fissuration dans des tôles minces.

- Fissuration par fatigue en traction ondulée $+F+0,1 F$, la valeur limite de l'interstrie est de l'ordre du micron.

6. Conclusions. - Dans le cas des éprouvettes que nous avons examinées et qui sont représentatives d'un revêtement d'avion, nous sommes arrivés aux conclusions suivantes, rassemblées dans le tableau II.

Dans les limites de vitesse définies ci-dessus, il est également possible :

- de remonter au nombre de cycles de propagation avec une bonne précision.

- de remonter à la contrainte pleine tôle avec une précision qui dépend de la distance à laquelle la mesure du $i_{\text {moy }}$ est faite :

$$
\begin{aligned}
\text { par exemple pour } a & =2 \mathrm{~mm} \delta \sigma= \pm 4 \mathrm{hb} \\
a & =10 \mathrm{~mm} \delta \sigma= \pm 2 \mathrm{hb} .
\end{aligned}
$$

En dehors des limites définies dans le tableau ci-dessus, il est encore possible dans la pratique d'apprécier avec suffisamment de garantie le nombre de cycles de propagation d'une fissure jusqu'au moment où l'on atteint la valeur asymptotique de $i_{\text {moy }}$ (en corrigeant en fonction des courbes d'étalonnage que nous avons tracées).

Mais un point important de cette étude sur alliage léger en faible épaisseur est qu'il ne semble pas y avoir d'interstrie supérieur à 1 ou $2 \mu$; passé ces valeurs macroscopiques de propagation de fissure de fatigue, il ne semble plus que la striation soit le phénomène réglant directement la vitesse de propagation. 